\title{
Body Mass Index and Dental Caries in Children
}

\author{
Zahra Bahrololoomi, ${ }^{1}$ Faezeh Fotouhi Ardakani, ${ }^{2}$ and Fahimeh Dehestani Ardakani ${ }^{3,}$ \\ ${ }^{1}$ Associate Professor, Member of Social Determinate of Oral Health Research Center, Pediatrics Department, Shahid Sadoughi University of Medical Sciences, Yazd, Iran \\ ${ }^{2}$ Assistant Professor, Department of Pediatrics Dentistry, Shahid Sadoughi University of Medical Sciences, Yazd, Iran \\ ${ }^{3}$ Dentist, Yazd, Iran \\ "Corresponding author: Fahimeh Dehestani Ardakani, Dentist, Yazd, Iran. E-mail: dehestani7of@gmail.com
}

Received 2016 June 28; Revised 2016 September 03; Accepted 2016 September 06.

\begin{abstract}
Background: Dental caries is the most common chronic childhood disease with serious implications for individual and public health. Obesity is the most common nutritional problem in children and caries are associated with a large number of negative health outcomes. Dental caries in children is reported to affects their anthropometric outcomes, but the evidence is conflicting.

Objectives: Based on the differences in the findings of the studies and the possible role of obesity in the pathogenesis of dental caries, the current study aimed to investigate the relationship between body mass index (BMI) and dental caries among children aged three to six years.

Methods: In this cross-sectional study, 400 children aged three to six years were selected through cluster sampling from Yazd kindergartens, Iran. Weight and height were measured using scale and tape measure, while decayed, missing, and filled teeth (DMFT) was recorded through intraoral examination using mirror, probe and flashlight. Then the relationship between calculated growth indices z-score (evaluated by world health organization (WHO) Anthro and WHO AnthroPlus software) and DMFT was considered. The results were analysed using SPSS ver. 20 and Kruskal-Wallis, Spearman correlation coefficient and Pearson tests.

Results: An inverse correlation was found between BMI $z$-score and DMFT $(\mathrm{P}=0.006, \mathrm{r}=-0.14)$. Assessment of the effect of demographic variables showed significant relationship in boys $(\mathrm{P}=0.01)$ and children under five years $(\mathrm{P}=0.04)$.

Conclusions: It appears that children with a high BMI z-score had lower DMFT and that there is a negative correlation between obesity and tooth decay.
\end{abstract}

Keywords: Dental Caries, Obesity, Body Mass Index, Child

\section{Background}

Weight gain and obesity, important indicators of health, are the most common nutritional disorders in children (1). The prevalence of obesity in children and adolescents is increasing rapidly among industrial and developing societies. In obesity, the body's excessive calories appear in the form of fat deposits, and this could have genetic or environmental aspects. Increased consumption of sweet beverages, lack of exercise, car use in all situations, and access to a variety of fast food have changed people's lifestyle (2). Body mass index (BMI), as the most common anthropometric method, is calculated by dividing weight in kilograms by height in squared metres (3). The z-scores were calculated using world health organization (WHO) Anthro software (available at: http://www.who.int/childgrowth/software/en/) and WHO AnthroPlus software (available from: http:/ www.who.int/growthref/en/) to elucidate the subject's status on the age- and gender-specific growth chart. The child who received two z-scores under the normal value $(<$ -2) was considered abnormal (4).

Dental caries is the most common oral infectious disease caused by multiple factors including increasing in- take of carbohydrate-rich food. Decayed, missing, and filled teeth (DMFT) are the most common evaluation indicators of dental caries showing the number of teeth decayed, missing, and filled (1). Dental problems in childhood affect communication and speaking skills, interfere with nutrition, have a negative impact on child growth and development (5).

Dental caries and obesity are associated with a great number of negative health outcomes (6). The role of nutrition in the maintenance of health and growth, as well as its relation to dental caries, is well known (4). Poor oral hygiene, low awareness among parents, high consumption of sweetened drinks and low socio-economic status are considered the main reasons for dental caries. Some of these factors are marked as risk factors associated with obesity (2).

The relationship between weight and oral health in children has controversial results (7). Studies such as those of Bahrololoomi et al. and Yao et al. showed a positive relationship between BMI and dental caries $(1,6)$. Some other studies such as those of Narksawat and Khosravani et al. showed a negative correlation between BMI and dental caries. Narksawat reported no correlation between oral 
hygiene and dental caries in the study population. Khosravani mentioned a significantly negative correlation coefficient between DMFT and BMI, whereas DMFT coincided with an increase in $\operatorname{BMI}(3,8)$. The studies by Sede MA and Edalat et al. cited no correlation between these two indices. Sede MA concluded that there was no statistically significant relationship between obesity, periodontal status and tooth decay experience in patients. Therefore, Edalat declared that there was no significant correlation between increasing DMFT and decreasing height, weight and BMI (4, 9).

\section{Objectives}

Due to the differences between studies and the risk of obesity in the pathogenesis of dental caries, the current study aimed to evaluate the relationship between BMI and tooth decay among children aged three to six years.

\section{Methods}

In this correlational and cross-sectional study, 400 children aged three to six years were selected through cluster sampling in Yazd city kindergartens, Iran. The sample size was calculated by considering $\alpha=0.05$ and $\mathrm{r}=0.2$ (correlation between DMFT and BMI z-score according to pilot study), based on the formula:

$C=0.5 \times \ln \left[\frac{(1+r)}{(1-r)}\right]$

$N=\left[\frac{\left(z_{\alpha}+z_{\beta}\right)}{C}\right]^{2}+3$

Therefore, the study was started by different areas in Yazd and identified kindergartens within each region as different clusters. Then a number of clusters was randomly selected from these clusters and finally sampled all the children of the kindergartens. The inclusion criteria in the study were: Healthy children with no systemic and physi$\mathrm{cal} / \mathrm{mental}$ disease in the age group of three to six years.

The exclusion criteria were: Uncooperative children, the presence of first permanent molar tooth, morphological abnormalities and tooth discoloration.

Intraoral examination was done to determine the DMFT index (number of decayed, missing teeth due to decay and filled primary teeth) by cleaning the kids' teeth with gauze using a mirror, a probe and a flashlight. Dental caries was determined visually with optimal illumination of the oral cavity, no X-rays were used. In the present study, national reference z-scores were applied to the study population under examination; therefore, weight (to the nearest $0.1 \mathrm{~kg}$ ) and height (to the nearest $5 \mathrm{~mm}$ ) measurements were taken with minimal clothing and no shoes using standard scale and tape measure. After that, the height and weight of each of the children were entered according to age and gender into the WHO Anthro (for 0 - 60 months children) and WHO AnthroPlus (WHO growth reference data for children aged 5 - 19 years) software to compare the data with those of the WHO standards for height and weight by age. This software estimates BMI index of children's health and fitness (10) in two groups: children under 60 months and children over that. Standard deviations from two of each indicator were considered abnormal. A cut-off of less than -2 safety datasheets (SDs) was used to report underweight and stunting, whereas a cutoff of more than +2 SDs was used to report obesity. Consequently, children were divided into three groups under -2 z-score, between -2 and 2, and over 2 (4). The relationship between DMFT index with weight for height z-score (WHZ), weight for age z-score (WAZ) and BMI for age z-score (BAZ) were determined. By coding the collected data, the results were finally analysed by the SPSS ver. 20, KruskalWallis test, Spearman correlation coefficient and Pearson test.

It should be noted that this article was approved by the ethics committee of Shahid Sadoughi University of Medical Science, Yazd, Iran, under the code: IR.SSU.REC.1394.51.

\section{Results}

Out of these 400 children, 229 were three-to-six-yearold boys. The mean DMFT of the study population was 3.75 \pm 3.75 , whereas only $27.2 \%$ of this population were caries free $(\mathrm{DMFT}=0)$. BMI distribution was as follows: $90.8 \%$ normal BMI, 5.2\% fat and 4\% had low BMI.

The mean of DMFT in children based on weight for height z-score (WHZ), weight for age z-score (WAZ), and BMI for age $\mathrm{z}$-score (BAZ) in three subgroups under - 2, between -2 and 2 , and over 2 respectively are shown in Table 1. There were no significant differences between these various groups of growth indices based on the Kruskal-Wallis test.

According to the Spearman correlation coefficient, the relationship between BAZ and WAZ with DMFT score was significantly reverse $(r=-0.14, \mathrm{P}=0.006),(\mathrm{r}=-0.17, \mathrm{P}=$ 0.001 ), which showed that with weight and body mass gain tooth decay became lower in children. Comparing the mean and median of DMFT based on different groups of the growth indices by age showed that mean DMFT score from Kruskal-Wallis analysis was not significant in these groups. The significant relationship between the mean DMFT score in boys based on WAZ groups $(\mathrm{P}<05)$ is shown in Table 2. 
Table 1. Comparison of the Mean and Median of DMFT Based on Study Groups ${ }^{\mathrm{a}, \mathrm{b}}$

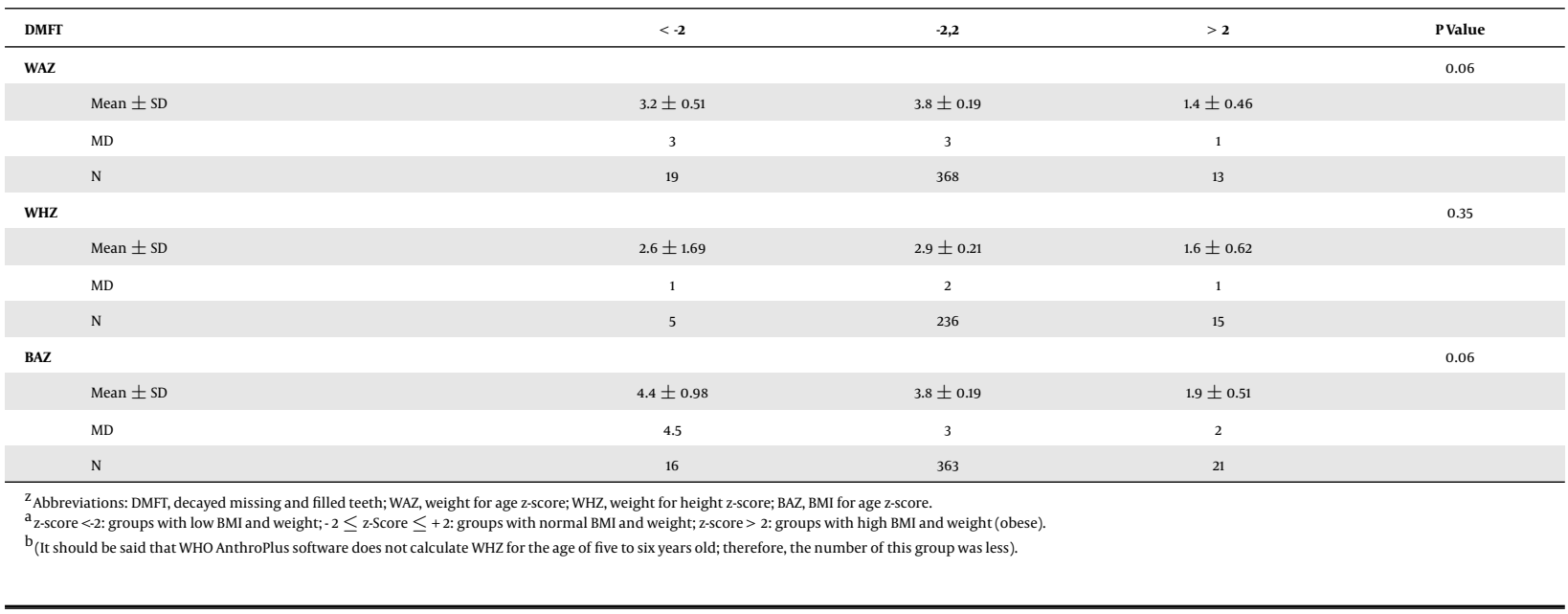

Table 2. Comparison of the Mean and Median of DMFT Based on Study Groups

\begin{tabular}{|c|c|c|c|c|}
\hline DMFT & $<-2$ & $-2,2$ & $>2$ & PValue \\
\hline \multicolumn{5}{|l|}{ WAZ } \\
\hline Boy & & & & $0.04^{*}$ \\
\hline Mean $\pm S D$ & $2.6 \pm 0.66$ & $4.1 \pm 0.26$ & $1.2 \pm 0.61$ & \\
\hline MD & 2.5 & 3 & 0.5 & \\
\hline Girl & & & & 0.25 \\
\hline Mean \pm SD & $4.1 \pm 0.70$ & $3.5 \pm 0.30$ & $1.6 \pm 0.74$ & \\
\hline MD & 5 & 3 & 2 & \\
\hline \multicolumn{5}{|l|}{ wHZ } \\
\hline Boy & & & & 0.25 \\
\hline Mean $\pm S D$ & $3 \pm 3$ & $3.1 \pm 0.29$ & $1.5 \pm 0.97$ & \\
\hline MD & 0 & 2 & 0 & \\
\hline Girl & & & & 0.95 \\
\hline Mean $\pm S D$ & $2 \pm 1$ & $2.7 \pm 0.32$ & $1.8 \pm 0.65$ & \\
\hline MD & 2 & 2 & 2 & \\
\hline \multicolumn{5}{|l|}{ BAZ } \\
\hline Boy & & & & 0.05 \\
\hline Mean $\pm S D$ & $5 \pm 1.21$ & $4 \pm 0.25$ & $1.7 \pm 0.86$ & \\
\hline MD & 6 & 3 & 0 & \\
\hline Girl & & & & 0.72 \\
\hline Mean $\pm S D$ & $2.5 \pm 1.32$ & $3.6 \pm 0.30$ & $2.2 \pm 0.57$ & \\
\hline MD & 2 & 3 & 2 & \\
\hline
\end{tabular}

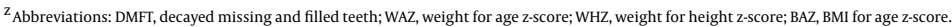

Table 3 shows DMFT and growth indices' correlation coefficient based on Pearson test by age and gender. WAZ and BAZ in boy and girl patients, WAZ in girl and WHZ and BAZ at the age of $\leq$ five years were significant.

The Spearman analysis showed a significant relationship between DMFT and BMI z-score $(\mathrm{P}=0.006)$. The correlation coefficient was negative $(r=-0.14)$, following an increase in the z-score, reduced DMFT.

\section{Discussion}

Oral health and nutrition have a bilateral relationship. While daily diet has an effect on oral health, oral health can play an important role in nutrition and general health status (11). For example, children with infected teeth and toothache run the risk of malnutrition, and this may be coupled with emaciation and arrested growth in height compared to those with unaffected teeth (12). Moreover, there are common factors such as sugary components, the 
Table 3. Determination of Correlation Coefficient of WAZ, WHZ and BAZ with DMFT Score Based on Age and Gender in the Study Subjects

\begin{tabular}{|c|c|c|c|c|c|c|}
\hline \multirow[t]{2}{*}{ DMFT/Variable } & \multicolumn{2}{|c|}{ WAZ } & \multicolumn{2}{|c|}{ WHZ } & \multicolumn{2}{|c|}{ BAZ } \\
\hline & PValue & $\mathbf{r}$ & P Value & $\mathbf{r}$ & P Value & $\mathbf{r}$ \\
\hline \multicolumn{7}{|l|}{ Gender } \\
\hline Boy & $0.008^{*}$ & -0.17 & 0.06 & -0.15 & $0.01^{*}$ & -0.16 \\
\hline Girl & $0.04^{*}$ & -0.15 & 0.23 & -0.11 & 0.11 & -0.12 \\
\hline$\leq 5$ & 0.08 & -0.10 & $0.03^{*}$ & -0.13 & $0.04^{*}$ & -0.12 \\
\hline$>5$ & 0.07 & -0.14 & - & - & 0.38 & -0.07 \\
\hline
\end{tabular}

${ }^{\mathrm{z}}$ Abbreviations: DMFT, decayed missing and filled teeth; WAZ, weight for age z-score; WHZ, weight for height z-score; BAZ, BMI for age z-score.

excessive consumption of which can lead to both obesity and dental caries (13). Taking into account the common risk factors of dental caries and growth indices in children, and the possible relationship between dental caries and physical development in children, the relationship between DMFT and growth indices of three-to-six-year-old children was conducted in the current study.

In this survey, children's body fitness was evaluated by the z-score of growth indices. One of the most accurate methods of BMI assessment in children is the application of the Anthro software designed by the WHO. It indicates children of both genders under 60 months and above (4).

Different studies reported conflicting results on the relationship between dental caries and growth indices. According to Yao et al. (2014), there is a positive association between obesity and dental caries. Furthermore, lifestyle choices and nutritional status are aggravating common factors of obesity and chronic diseases such as dental caries (6). Sede et al. did not find any significant correlation between obesity and tooth decay in their study. They stated that education and good socio-economic status have a positive impact on children's quality of life and eliminates the risk of chronic diseases such as obesity and tooth decay (9). This difference in findings could be due to racial differences, complexity, as well as the factors of obesity and tooth decay. It means that a number of factors such as environment, gene, and behaviour may have a role in the occurrence of these two problems (5).

In the current study, the obtained inverse correlation between growth indices WAZ, BAZ, and DMFT showed that an increase in weight and body mass reduced DMFT. In other words, there was higher tooth decay in the subjects with lower weight. This inverse relationship can be observed in other studies such as those of Lempert et al., Koksal, and Sheiham (14-16). Lempert et al. mentioned that this inverse association might be that such severe dental caries especially in young children may affect growth in an ad- verse manner, although this may be applied only to height and not BMI (15).

According to Cameron et al., being underweight in children with established dental decay could be due to the inability of chewing, which in turn can affect their food choice (12). Research performed on the nutritional status of children with severe caries showed that dental caries is a risk factor for malnutrition as it triggers loss of nutrition and BMI for age (BAZ), thereby causing a significant health problem (15). Sheiham et al. made three major statements in this regard: First, untreated caries associated infection can cause pain and discomfort, leading to insufficient food intake of painful eating; second, severe caries can affect children's quality of life and consequently their growth. The implications include pain, irritability and disturbed sleep. Disturbed sleep may adversely affect glucosteroid production and growth. Although not all untreated dental caries affect general health, they have a significant impact on children's quality of life and their dietary intake. The third possible mechanism of how untreated severe caries with pulpitis can affect growth is that chronic inflammation due to pulpitis and dental abscesses affects stimulation of metabolic pathways and production of cytokines such as interleukin-1(IL-1), leading to erythropoiesis inhibition. This and the suppression of haemoglobin production can lead to anaemia, turning the child underweight (16).

In the current study, lower DMFT in girls and boys under five years could be related to oral health education programmes available for mothers in child health care centres during pregnancy, infancy, breastfeeding and childhood periods. Moreover, parents need to pay more attention to children's nutrition because children's access to snacks and fast food may be the reason for BMI increase in this age group.

Also, limitations should be noted: Both overweight/ obesity and caries are conditions with multifactorial causes which can be influenced by several factors. 
The study observed that with increasing awareness on the part of parents about oral hygiene and prevention of dental diseases in children with timely dental treatment, children's quality of life, general growth and development can be ensured.

In terms of future research, longitudinal studies are needed to determine whether there is a cause-and-effect relationship between caries levels and poor growth. Such a study would benefit from adequate adjustment to confound variables and from the use of continuous outcomes in addition to categories of malnutrition.

\subsection{Conclusion}

Owing to the limitations of the study, the results showed a negative relationship between increasing growth indices and dental caries. As a result, children with high body weight and BMI showed a low DMFT index.

Future longitudinal studies can help determine whether there is a cause-and-effect relationship between caries levels and poor growth.

\section{References}

1. Bahrololoomi Z, Soruri M, Rekabi M, Ravaei S. The Relationship between BMI and DMFT/dmft among 7-11 Year-old Children in Yazd. J Shahid Sadoughi Univ Med Sci. 2014;21(6):751-8.

2. Faezi M, Jalayer N, Lashkari S. Determination of association between some caries-related factors in permanent teeth with body mass index of female school students in tehran, iran. 2013

3. Khosravani SR, Golkari A, Memarpour M. Assessing the Relationship between Dental Caries and Anthropometric Indices in 9-11Year-old Primary School Children of Shiraz. Armaghane Danesh. 2014;18(10):787-69.

4. Edalat A, Abbaszadeh M, Eesvandi M, Heidari A. The relationship of severe early childhood caries and body mass index in a group of 3-to 6-year-old children in Shiraz. J Dent. 2014;15(2):68.
5. Mohtadinia J, Ejtahad H, Parizan S, Kalejahi PN. The relationship between dental caries and body mass index and food habits in children referred to dentistry clinic of Tabriz University of medical sciences. Yafteh. 2011;12(3):71-8.

6. Yao Y, Ren X, Song X, He L, Jin Y, Chen Y, et al. The relationship between dental caries and obesity among primary school children aged 5 to 14 years. Nutr Hosp. 2014;30(1):60-5. doi: 10.3305/nh.2014.30.1.7552. [PubMed: 25137263].

7. Shahraki T, Shahraki M, Omrani Mehr S. Association between body mass index and caries frequency among zahedan elementary school children. Int J High Risk Behav Addict. 2013;2(3):122-5. doi: 10.5812/ijhrba.10220. [PubMed: 24971289].

8. Narksawat K, Tonmukayakul U, Boonthum A. Association between nutritional status and dental caries in permanent dentition among primary schoolchildren aged 12-14 years, Thailand. Southeast Asian J Trop Med Public Health. 2009;40(2):338-44. [PubMed:19323020].

9. Sede MA, Ehizele AO. Relationship between obesity and oral diseases. Niger J Clin Pract. 2014;17(6):683-90. doi: 10.4103/1119-3077.144378. [PubMed: 25385902].

10. World Health Organization. Growth reference data for 5-19 years 2011. Available from: http://www.who.int/childgrowth/software/en/.

11. Tramini P, Molinari N, Tentscher M, Demattei C, Schulte AG. Association between caries experience and body mass index in 12-year-old French children. Caries Res. 2009;43(6):468-73. doi: 10.1159/000264684. [PubMed: 20016177].

12. Cameron FL, Weaver LT, Wright CM, Welbury RR. Dietary and social characteristics of children with severe tooth decay. Scott Med J. 2006;51(3):26-9. [PubMed:16910048].

13. Zero DT, Fontana M, Martínez-Mier EA, Ferreira-Zandona A, Ando M, González-Cabezas C, et al. The biology, prevention, diagnosis and treatment of dental caries: scientific advances in the United States. JAm Dent Assoc. 2009;140:25S-34S.

14. Koksal E, Tekcicek M, Yalcin SS, Tugrul B, Yalcin S, Pekcan G. Association between anthropometric measurements and dental caries in Turkish school children. Cent Eur J Public Health. 2011;19(3):147-51. [PubMed: 22026291].

15. Lempert SM, Froberg K, Christensen LB, Kristensen PL, Heitmann BL. Association between body mass index and caries among children and adolescents. Community Dent Oral Epidemiol. 2014;42(1):53-60. doi: 10.1111/cdoe.12055. [PubMed: 23763718].

16. Sheiham A. Dental caries affects body weight, growth and quality of life in pre-school children. Br Dent J. 2006;201(10):625-6. doi: 10.1038/sj.bdj.4814259. [PubMed: 17128231]. 